\title{
Modeling of Neem Oil Methyl Esters Production using Artificial Neural Networks
}

\author{
Y.C. Bhattacharyulu, \\ $\mathrm{PhD}$ \\ Senior Prof., Dept. of \\ Chemical Engineering, \\ Anuradha Engineering \\ College, Chikli.
}

\author{
V. N. Ganvir \\ Asst. Prof., Dept. of \\ Petrochemical \\ Technology, \\ Laxminarayan Institute of \\ Technology, Nagpur
}

\author{
Aditaya Akheramka \\ (B.Tech) Dept. of \\ Petrochemical \\ Technology, \\ Laxminarayan Institute \\ of Technology, Nagpur
}

\author{
Amol Ramning \\ (M. Tech) Dept. of \\ Petrochemical \\ Technology, \\ Laxminarayan Institute \\ of Technology, Nagpur
}

\begin{abstract}
The objective of the present work is to develop models inculcating the effect of operating conditions of neem oil methyl esters (NOME) production in an oscillatory baffled reactor, namely temperature, time of reaction, oil to methanol ratio and catalyst concentration on the estimation of parameters like the viscosity of biodiesel produced by using Artificial Neural Networks technique. Experiments were conducted in the laboratory and the results obtained were used to develop the ANN model using MATLAB. The developed model was in good agreement with the experimental values (error within $\pm 1 \%$ ). Based on the outcome of this demonstrative work, it can be concluded that ANN has a great potential in addressing the estimation of biodiesel properties. It is sincerely felt that the methodology adopted in the present work can be extended to more comprehensive data sets and various data from different experimental reactor design setups.
\end{abstract}

\section{Keywords}

Neem Oil Methyl Ester, Oscillatory baffled reactor, artificial neural network.

\section{INTRODUCTION}

The depletion of easy petroleum reserves, the instability of petroleum sources, recent increase in petroleum prices and uncertainties concerning petroleum availability are a few of the reasons for the increase in the interest in vegetable oil fuels. Vegetable oils hold promise as alternative fuels for

diesel engines. But undesirable properties like high viscosities, low volatilities and poor cold flow properties have led to the development of various derivatives. Out of these, fatty acid methyl esters, derived from triglycerides by transesterification with methanol, known as biodiesel, have received the most attention.

Biodiesel is a liquid fuel similar to petroleum diesel in combustion properties but is essentially free of sulfur, making it a cleaner burning fuel than petroleum based diesel. Biodiesel is a promising non-toxic and biodegradable renewable fuel comprising of mono-alkyl esters of long chain fatty acids, which is produced by a catalytic trans esterification reaction of vegetable oils with short chain alcohols. It has become an interesting alternative to diesel, because it has similar properties to the traditional fossil diesel fuel and may thus substitute diesel fuel with none or very minor engine modifications. It is proved to be non-toxic and eco-friendly, leading to lower emissions of $\mathrm{SOx}, \mathrm{CO}$, unburnt hydrocarbons and particulate matter.

Mostly biodiesel is prepared from edible oils such as soybean, rapeseed, palm; few attempts have been made using non edible oils such as neem oil, karanja, babsuu, jatropa and tobacco seed oil. The reaction is usually carried out using methanol and an alkaline catalyst $(\mathrm{NaOH}, \mathrm{NaOMe})$ in the temperature range $60-80{ }^{\circ} \mathrm{C}$.

The production of biodiesel is greatly increasing due to its environmental benefits. However, production costs are still rather high compared to petroleum based diesel fuel. Also, direct application of vegetable oil as fuel to diesel engines is not possible due to its higher viscosity. Hence reduction of vegetable oil viscosity is an urgent need. The viscosity of vegetable oils can be reduced by using different methods namely blending, pyrolysis, micro-emulsification and trans esterification. Trans -esterification methods are most widely used to reduce the viscosity and improve the fuel properties of vegetable oils. But the trans-esterification reaction is strongly influenced by several factors such as molar ratio of alcohol, catalyst concentration, free- fatty acid in oil samples, reaction temperature and time.

The present work aims to develop models inculcating the effect of these operating conditions, namely temperature, time of reaction, oil to methanol ratio and catalyst concentration as independent parameters. These independent parameters are used in estimation of dependent parameters like the viscosity of biodiesel produced. The correlation technique adopted in the present work is Artificial Neural Networks.

\subsection{Application of Artificial Neural Network to Biodiesel processes}

Artificial Neural Network (ANN), a popular modeling tool for processes where nonlinear multivariable relationships are involved, is also called as black box modeling and its working principle is loosely modeled on the biological neural network. It is composed of a large number of data processing elements called as nodes or neurons arranged in layers and interconnected with each other to develop a correlation [1].

Multi-Layer Perception (MLP) is the most common type of ANN employed in modeling of chemical processes. It is a feed forward error back propagation neural network and consists of input and output layers apart from at least one hidden layer in between them. The number of nodes in input and output layers is decided by the number of independent and dependent parameters that define the process. The number of hidden layers and the number of nodes in each hidden layer 
are dependent upon the complexity of the model, the number of input and output nodes and, the data sets involved. Nodes in successive layers are connected to each other by means of constants called as weights. The outputs from the input layer are fed to hidden layer units, which in turn, feed their outputs to the next hidden layer nodes. The training of MLP is usually carried by error back propagation algorithm suggested by Rumelhart [2].

In the following paragraphs a brief account of application of ANN to biodiesel processes are reviewed.

Roman M. Balabin et al analyzed biodiesel quality using near infrared spectroscopy technique in terms of biodiesel properties like density, kinematic viscosity, methanol and water content employing ANN modeling and claimed ANN approach was superior to the linear (MLR, PCR, PLS) and “quasi"'-non-linear (Poly-PLS, Spline-PLS) calibration methods. The authors compared the performance of linear and non-linear calibration techniques - namely, multiple linear regression (MLR), principal component regression (PCR), partial least squares regression (PLS), polynomial and SplinePLS versions, and artificial neural networks (ANN) - for prediction of biodiesel properties from near infrared spectra. The model was created for four important biodiesel properties, namely: density, kinematic viscosity, water content, and methanol content. The developed model prediction capability was also accessed by the authors employing Savitzky-Golay derivatives and orthogonal signal correction methods [3].

Machavaram Rajendra and coworkers developed ANN coupled with genetic algorithm on MATLAB platform for predicting the optimized biodiesel process parameters in reducing high FFA vegetable oils suitable for transesterfication method. They used feed forward back propagation network with one input, two hidden and one output layers, in predicting the acid value of biodiesel with feed acid value, methanol to oil ratio, catalyst concentration and reaction time. The feed stocks studied were jatropha, mahua, simaruoba and rice bran oils [4].

Liew Weng Hui et al used ANN to predict the dynamic trending for 10 reaction components based on the input parameter of time. The ANN used is feed-forward type with single hidden layer and coupled with Levenberg-Marquardt (LM) training algorithm. The authors proposed a new method to account for damping factor in LM algorithm, which reduced the number of epochs, thus improved performance in terms of time against default method. They concluded that the number of epochs is related to the value of updating factor and ANN has great potential for application of biodiesel processes modeling in improving the performance of biodiesel production methods [5].

Feasibility studies of biodiesel production plant were investigated by Fahmi and Cremschi using ANN as the surrogate models. The model was tested in a process simulator, and the resulting cost of the simulation studies differs only by about $1 \%$, in predicting the accuracy by ANN, and the authors concluded that ANN requires large amount data as training which involves cost [6]. Sabuncuoglu and Seung-Soo in their work said that among various surrogate models ANN has proved to be a powerful tool both for complexity reduction and accurate mapping-function modeling $[7,8]$.
Ignition quality is one of the important properties of biodiesel that is determined by the composition of the FAME components which depends on the nature of the feed stock used for production of biodiesel by transesterfication reaction. Cetane Number $(\mathrm{CN})$ is a commonly used indicator for the determination of diesel fuel quality, especially the ignition quality. Four types of ANN models to predict the cetane number of biodiesel used were multi-layer feed forward (MLFFN), radial base function (RBFN), generalized regression (GRNN), and recurrent network (RNN). The fatty acid composition in percentage of various esters in pure form as well as mixtures of esters (biodiesel) and their experimental $\mathrm{CN}$ were taken into account for developing the models. The least error was obtained for MLFF network and recurrent network. Hassan Ghorbanil et al outlined modern trends in modeling the process together with their scope and range of application have been detailed. [9]. Kumar et al. in another study selected the best ANN for estimating properties of diesel-biodiesel blends [10].

Data collected from literature on biodiesel properties such as viscosity, flash point, high-heating values has been used by Kraipat Cheenkachorn to correlate fatty acid composition of various vegetable oils with biodiesel properties by ANN. Fatty acids such as palmitic acid, stearic acid acid, oleic acid, were used in their study. The predicted properties include viscosity, high-heating value), and cetane number. The results show that both statistical models and the artificial neural networks precisely predict the properties of biodiesel derived from certain vegetable oils. The authors claim that predicted values were very close to the experimental results [11].

Blends of ethanol, bio-ethanol, diesel and biodiesel were made for studying fuel properties such as water and sediment, dynamic viscosity, kinematic viscosity and density. Experimental data were analyzed by means of Matlab software. The results obtained from artificial neural network analysis of the data showed that the network with feed forward back propagation of the Venberg-Marquardt train LM function with 10 neurons in the hidden layer was the best for predicting the parameters. The experimental data had a good correlation with ANN-predicted values according to authors with regression coefficient 0.96448 [12].

The spectral width in a near infra red spectrum of $9000 \mathrm{~cm}^{-1}$ (near-IR spectrum ranges from $4000-13000 \mathrm{~cm}^{-1}$ ) is divided into several small band widths called points or variables in order to find the spectrum of samples by the scanning instruments. For example a $16 \mathrm{~cm}^{-1}$ band width will yield 1125 spectral points or also called spectral variables, makes the sample analysis unreliable due to this large number. Various techniques are available to reduce the number of wave length points to make the model predictable ability better. Feature selection or wave length selection is a critical step in spectral analysis methods. Roman M. Balabina et al compared the performance of sixteen different feature selection methods [13]. Their influence on the accuracy of biodiesel analysis was evaluated, and the prediction of four important parameters of biodiesel quality, namely density, viscosity, methanol content, and water content, was discussed. Two linear techniques for calibration model building, namely multiple linear regression (MLR) and partial least squares regression/projection to latent structures (PLS), are used for the evaluation of biodiesel properties based on near-infrared spectroscopy (NIRS) data. A comparison with a non-linear calibration model, artificial neural networks (ANN) or support vector machine (SVM), was also provided. 
M Rajendra et al developed an ANN program coupled with GA to predict optimized parameters in the pretreatment process of high FFA vegetable oils to reduce its acid content suitable for transesterficaion reaction on a MATLAB platform.

The developed ANN was a feed forward back propagation network with one input, two hidden and one output layers. The input parameters for the ANN to generalize the pretreatment process were initial acid value of vegetable oil ,methanol-to-oil ratio, catalyst concentration and reaction time and the output parameter was final acid value of oil. The developed ANN was trained with the experimental data obtained for jatropha, mahua, simaruoba and rice bran oils with acid value more than $14 \mathrm{mg} \mathrm{KOH/g-oil} \mathrm{[14].}$

Efforts have also been made by researchers to use the intelligence tools, and in particular Artificial Neural Network, to develop predictive models for the determination of biodiesel related properties. These include application of modeling techniques for prediction and optimization of biodiesel production processes [15], application of artificial neural network to predict properties of diesel -biodiesel blends [16], etc.

Hassan Ghorbani et al discussed the various modeling techniques in predicting and optimization of biodiesel processes concluded that ANN was a powerful tool to find the optimal parameters to produce biodiesel from different feed oil feed stocks, and also capable of process modeling, and aids in improving the conversion by neural network analysis. The authors conclude that mathematical models are not always effective due to their intrinsic drawbacks and limitations, but ANN has been proved to be an option in detecting the complex nonlinear processes [15]

\section{MATERIALS AND METHODS FOR NEEM OIL METHYL ESTER PRODUCTION}

\subsection{Materials}

The Neem oil used in this present study was obtained from Neem Foundation, Nagpur, India. All the chemicals used were of analytical reagent grades. The crude neem oil was unrefined but filtered, and the appearance of oil was dark brown in color.

The acid value of crude Neem oil was found to be $10.92 \mathrm{mg}$ $\mathrm{KOH} / \mathrm{gm}$ of oil sample. If the acid value of the oil is less than 1.0 , single stage transterification process is employed to convert the free fatty acid (FFA) content of the oil to the methyl ester. But higher FFA oils require a pre-treatment step called esterification to bring down the FFA content of oil to less than 1. Since Neem oil has an acid value of 10.92 which means it has higher FFA content, the oil requires a pretreatment step, before transesterfication. The free fatty acids were first converted to ester in a pretreatment process using methanol as a reagent in presence of sulfuric acid as a catalyst, with a volume to volume $(\mathrm{v} / \mathrm{v})$ ratio of $1-5 \%$ to reduce the acid value of Neem oil near to $2 \mathrm{mg} \mathrm{KOH} / \mathrm{gm}$. The next process is the transterification, where tri acylglycerol present in treated oil is converted to methyl ester using methanol as a reagent and $\mathrm{KOH}$ as an alkaline catalyst.

\subsection{Procedure}

\subsubsection{Pre- treatment}

In the pretreatment process, $100 \mathrm{ml}$ of Neem oil was mixed with methanol and $1 \%-5 \%$ sulfuric acid in a $300 \mathrm{ml}$ oscillatory baffled reactor. The reactor is maintained at room temperature $\left(25^{\circ} \mathrm{C}\right)$ throughout the reaction. The reaction and mixing was carried out under oscillatory baffled agitation in the reactor. Then, the mixture was allowed to settle in a conical bottomed separating funnel for 10 hours, resulted in two layers, with the top layer contains fatty acid methyl ester (FAME) and untreated glycerides. This layer was then subjected to transterification process. The bottom layer contains water and fatty acids and were separated from the upper layer [17].

\subsubsection{Transterification}

The transterification process was carried out in a $300 \mathrm{ml}$ oscillatory baffled reactor. Required quantity of $\mathrm{KOH}$ catalyst is dissolved in methanol with $80 \mathrm{ml}$ of treated oil obtained in first step was added to the reactor. The reaction was carried out at room temperature under continues oscillatory baffled agitation. The mixture was allowed to settle in a conical bottomed separating funnel. After 10 hours of settling the reaction mixture was separated into two layers, the upper layer being , Fatty acid methyl esters (FAME or Biodiesel) and the lower layer contains mostly glycerol.

\subsubsection{Analysis}

Viscosity of biodiesel samples were measured as per ASTM standards.

\section{DEVELOPMENT OF ARTIFICIAL NEURAL NETWORK MODEL}

In the present work the data related to the experiments carried out in the laboratory for the production of biodiesel from neem oil has been used for modeling purposes. A consolidated data set comprising of 30 data sets is compiled and parameters like the temperature at which the reaction is carried out, the time of reaction in hours, the catalyst concentration as weight percent and the oil to methanol ratio have been used as the independent input parameters. The models developed are used for the prediction of the one dependent parameter, namely the viscosity of biodiesel produced in each of the 30 independent runs.

ANN model is developed using MATLAB R2008b, inculcating the parameters of the present work. It contains of one hidden layer consisting of 20 neurons. The architectures of the topology for ANN model is depicted in the Fig 01. 


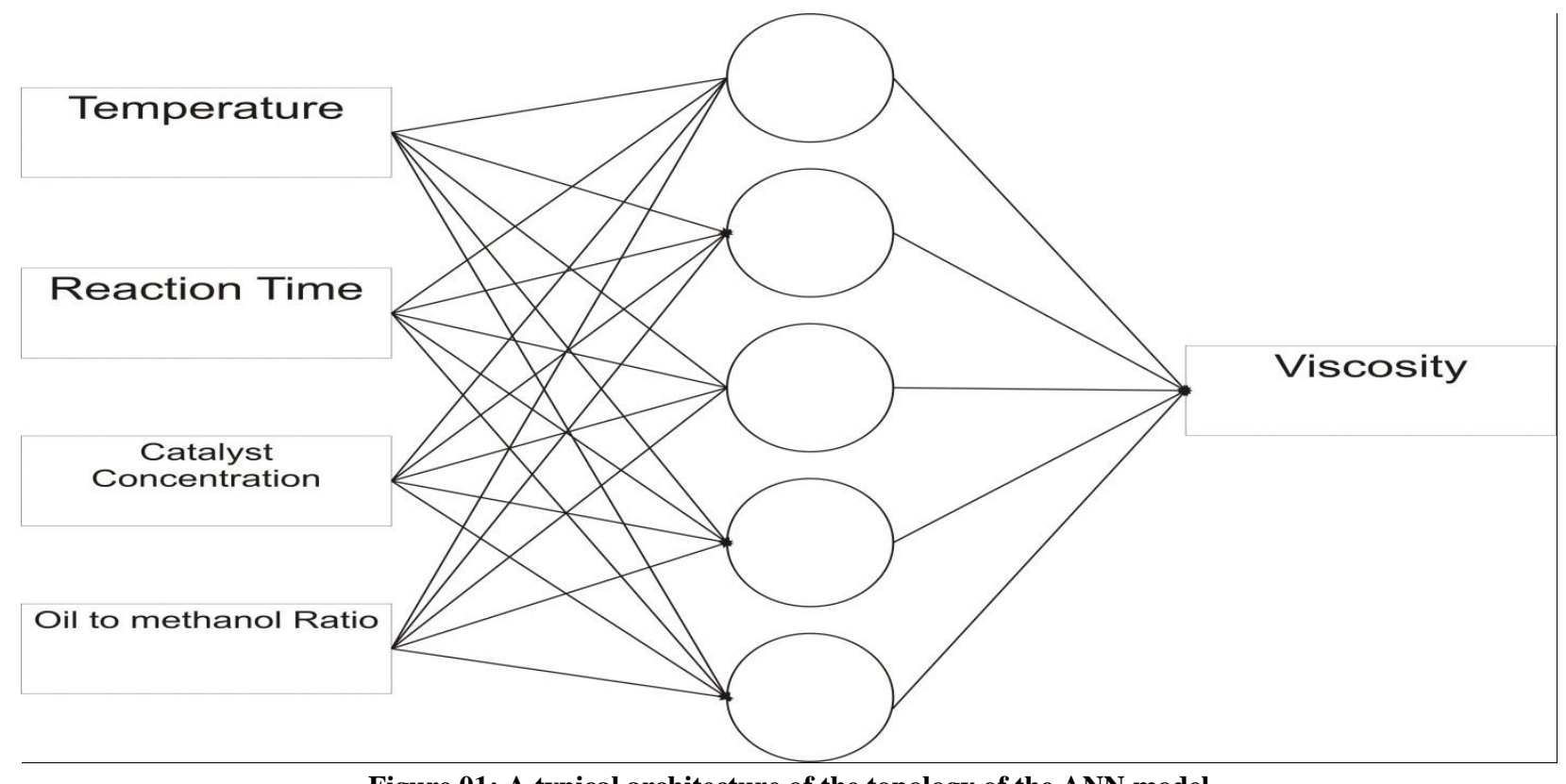

Figure 01: A typical architecture of the topology of the ANN model

\section{RESULTS AND DISCUSSION:}

Total data set of 30 points has been used in developing ANN model using MATLAB R2008b. The Fig. 02 shows the Mean Square Errors (MSE) for all the values under training, testing and validation. The $\mathrm{x}$-axis shows the number of iterations and the y-axis shows the MSE values. The regression coefficients of the training, testing, validation and the overall model developed using ANN is shown in Fig. 03 to Fig. 06. The values on the $\mathrm{x}$-axis are the target values or the experimental values input to develop the model whereas the values on the $\mathrm{y}$-axis are the values predicted by the ANN model developed. As can been seen from these high regression values the values predicted are very close to the actual viscosity values for all the data sets \& indicative of the successful development of the ANN model developed. 


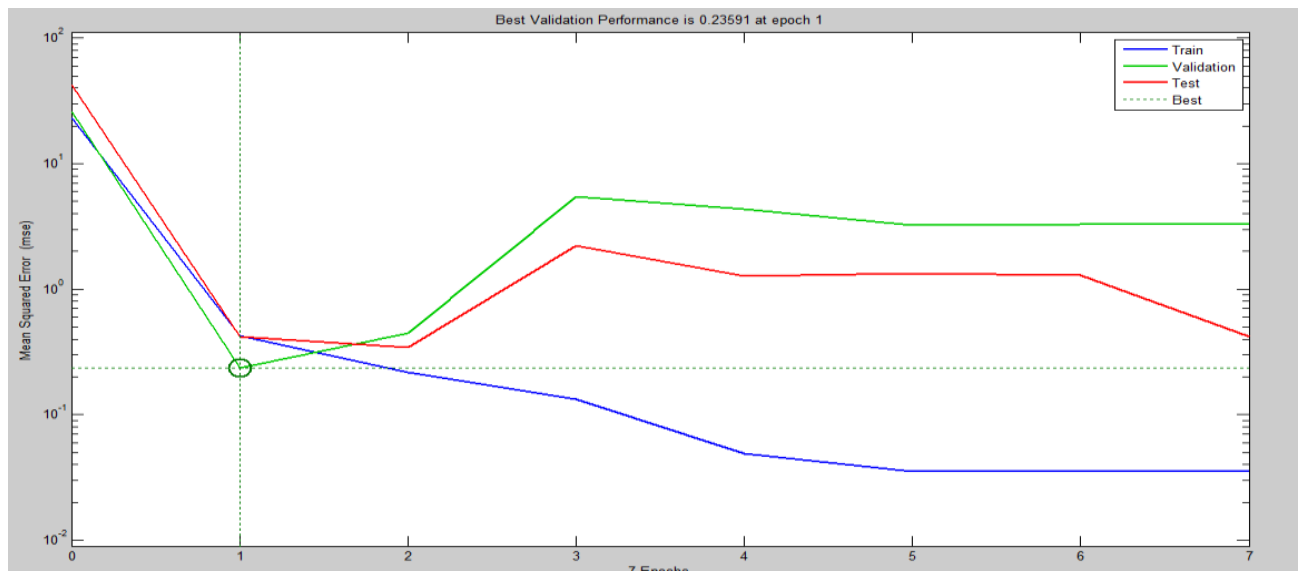

Figure 02: RMSE for training, testing and validation data for the ANN model developed

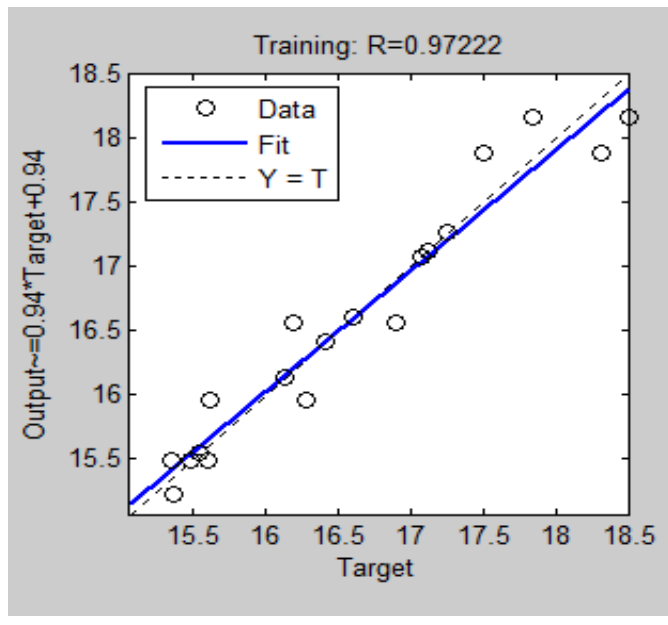

Figure 03: Regression values for the training data

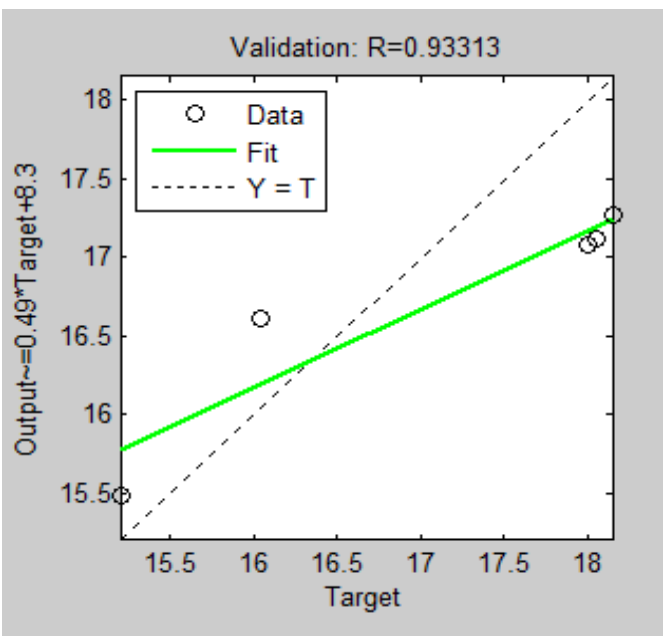

Figure 05: Regression values for the validation data

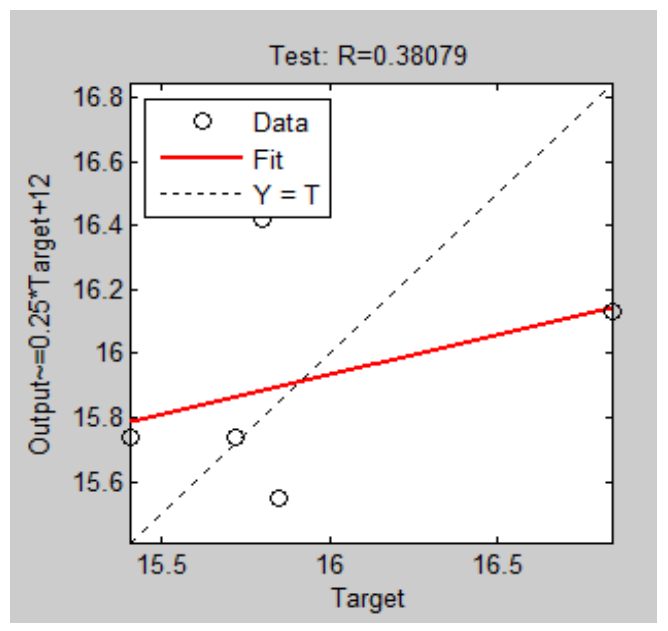

Figure 04: Regression values for the test data

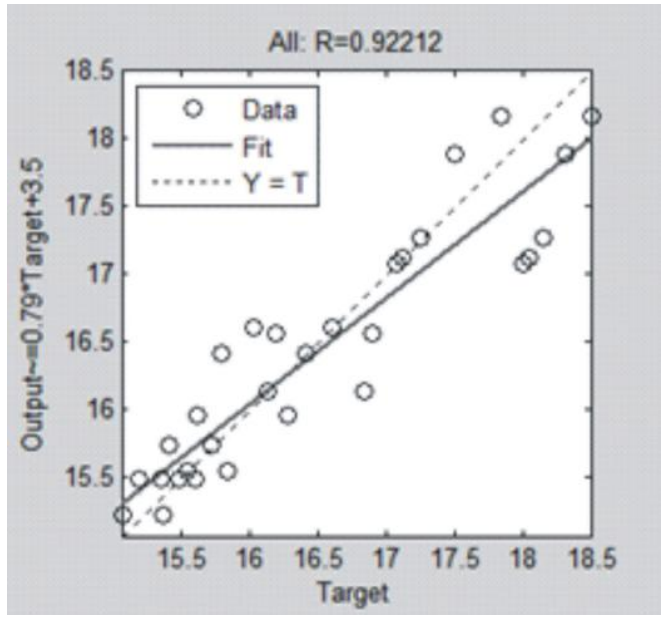

Figure 06: Regression values for the overall model

\section{CONCLUSION}

The recent uncertainty in the production of crude oil worldwide has triggered the increased interest of researchers 
in the development of fuels from natural sources, particularly fatty acid methyl esters, derived from triglycerides by trans esterification with methanol, known as biodiesel, have received the most attention. The objective of the present work is to correlate the effect of the operating parameters on the viscosity of biodiesel produced in an Oscillatory Baffled Reactor using Artificial Neural Networks as a modeling tool. It can be seen that the comparison of the viscosity of biodiesel is well in acceptable limits. Also, the percentage error for all the data points is under acceptable limits. The present work is demonstrative \& based on the outcome it can be concluded that ANN has great potential in addressing to the estimation problems related to yields incorporating the operating parameters of biodiesel production and should be extended to more comprehensive data sets. This would save on the time and energy and increase the accuracy of the estimations.

\section{ACKNOWLEDGEMENT}

The authors would like to express their sincere gratitude to Dr. S. D. Dawande, Director- Laxminarayan Institute of Technology for providing facilities to conduct practical work in the Petrochemical Technology laboratory, Laxminarayan Institute of Technology, Nagpur.

\section{REFERENCES}

[1] Anderson J.A, An Introduction to Neural Networks Prentice-Hall of India, Pvt Ltd New Delhi, (1999).

[2] Rumelhart D E \& McClleland Back Propagation Training Algorithm Processing, M.I.T Press, Cambridge Massachusetts, (1986).

[3] Roman M. Balabin, Ekaterina I. Lomakina, Ravilya Z. Safieva Neural network (ANN) approach to biodiesel analysis: Analysis of biodiesel density, kinematic viscosity, methanol and water contents using near infrared (NIR) spectroscopy, Fuel 90 (2011) 2007-2015.

[4] Machavaram Rajendra; Prakash Chandra Jena; Hifjur Raheman, Prediction of optimized pretreatment process parameters for biodiesel production using ANN and GA Fuel (May 2009), 88 (5), pg. 868-875.

[5] Liew Weng Hui, Zahira Yaakob, Siti Rozai mah Sheikh Abdullah, Artificial Neural Network Modeling and Performance Optimization on Biodiesel Production Process, Journal of Applied Sciences Research, 8(9): 4854-4864, 2012.

[6] Fahmi.I and Cremschi.S, University of Tulsa, Process Synthesis of Biodiesel Production Plant Using Artificial Neural Networks As the Surrogate Models, paper presented at Annual Meeting 2011 AIChE,10-20.2011.
[7] H. Seung-Soo and G. S. May, "Using neural network process models to perform PECVD silicon dioxide recipe synthesis via genetic algorithms," Semiconductor Manufacturing, IEEE Transactions on, vol. 10, pp. 279287, 1997.

[8] I. Sabuncuoglu and S. Touhami, "Simulation meta modeling with neural networks: an experimental investigation," International Journal of Production Research, vol. 40, pp. 2483-2505, 2002

[9] Hassan Ghorbani1, Ali M.Nikbakht2, Meisam Tabatabaei3, Mahdi Hosseini1+, Poya Mohammadi1, Application of modeling techniques for prediction and optimization of biodiesel production processes, 2011 International Conference on Biotechnology and Environment Management IPCBEE vol.18 (2011)IACSIT Press, Singapoore.

[10] J. Kumar, A. Bansal., Selection of Best Neural Network for Estimating Properties of Diesel-Biodiesel Blends. Greece, February. 2007, 16-19.

[11] Kraipat Cheenkachorn, Predicting Properties of Biodiesels Using Statistical Models and Artificial Neural Networks, As. J. Energy Env. 2006, 7(02), 299-306.

[12] P. Nematizade, B. Ghobadian and G. Najafi, Investigation of fossil fuel and liquid biofuel blend properties using artificial neural network, International Journal of Automotive and Mechanical Engineering (IJAME); ISSN: 2180-1606 (Online); Volume 5, pp. 639-647, January-June 2012 OUniversiti Malaysia Pahang.

[13] Roman M. Balabina, Sergey V. Smirnovb, Variable selection in near-infrared Spectroscopy: Benchmarking of feature selection methods on biodiesel data, Analytica Chimica Acta 692 (2011) 63-72.

[14] Prediction of optimized pretreatment process parameters for biodiesel production using ANN and GA , Machavaram Rajendra; $\quad$ Prakash Chandra Jena; Hifjur Raheman Fuel (May 2009), 88 (5), pg. 868-875.

[15] G Hassan, Ali M.Nikbakht, MeisamTabatabaei, Mahdi Hosseini, Poya Mohammadi, International Conference on Biotechnology and Environment Management, IPCBEE Vol.18 (2011), IACSIT Press, Singapore.

[16] Jatinder Kumar, Ajay Bansal, Kathmandu University Journal of Science, Engineering and Technology,Vol. 6, No.II, November, 2010, pg 98-103.

[17] Anindita Karmarkar, Prasanta Kumar Biswas, Souti Mukherjee. Environment-Congenial biodiesel Production from Non- Edible Neem oil. Environ Eng. Res. 2012 December, 17(s1):S27-S32. 